\title{
El modelo ecológico de Bronfenbrenner-Morris como marco explicativo de los factores que inciden en la vacunación versus COVID-19.
}

\section{The ecological model of Bronfenbrenner-Morris as an explanatory framework of the factors that influence vaccination versus COVID-19.}

\author{
Juan L. Arrieta-Villarreal ${ }^{a}$, Rebeca Guzmán-Saldaña ${ }^{b}$, Abel Lerma-Talamantes $^{c}$
}

\begin{abstract}
:
The ecological model of Bronfenbrenner-Morris (1998) allows studying the biopsychosocial factors associated with vaccination against COVID-19. This model facilitates the identification and understanding of all the variables that directly and indirectly affect the person, and that are decisive factors for individuals to be vaccinated or not; It should be noted that some of these factors are not even considered when it comes to promoting actions through vaccination campaigns. In Figure 1, the four subsystems defined in the model can be observed, with their elements that make them up: a) Microsystem (e.g, family experiences and changes in daily routine), b) Mesosystem (e.g., vaccination locations and the location of the person), c) Exosystem (e.g., social networks and the media), and d) Macrosystem (e.g., the anti-vaccine movement and the called "Conspiracy Theories").
\end{abstract}

\section{Keywords:}

Bronfenbrenner-Morris Model; Vaccination; COVID-19.

\section{Resumen:}

El modelo ecológico de Bronfenbrenner-Morris (1998) permite estudiar los factores biopsicosociales asociados con la vacunación contra la COVID-19. Este modelo facilita la identificación y comprensión de todas las variables que inciden directa e indirectamente sobre la persona, y que son factores decisivos para que los individuos se vacunen o no; cabe señalar que algunos de estos factores incluso ni se consideran cuando se trata de promover acciones a través de las campañas de vacunación. En la Figura 1 se pueden observar los cuatro subsistemas definidos en el modelo, con sus respectivos elementos que los integran: a) Microsistema (p. ej. experiencias familiares y cambios en la rutina cotidiana), b) Mesosistema (p. ej. campañas de vacunación locales y la ubicación de la persona), c) Exosistema (p.ej. redes sociales y los medios de comunicación), y d) Macrosistema (p.ej. el movimiento antivacunas y las llamadas "Teorías Conspirativas").

\section{Palabras Clave:}

Modelo Bronfenbrenner- Morris; Vacunación; COVID-19.

\footnotetext{
${ }^{a}$ Autor de Correspondencia, Universidad Autónoma del Estado de Hidalgo, https://orcid.org/0000-0003-0086-7678,Email: ar295077@uaeh.edu.mx

${ }^{b}$ Universidad Autónoma del Estado de Hidalgo, https://orcid.org/0000-0003-0877-4871, Email: rguzman@uaeh.edu.mx

${ }^{c}$ Universidad Autónoma del Estado de Hidalgo, https://orcid.org/0000-0001-7212-641X, Email: abel_lerma@uaeh.edu.mx
} 


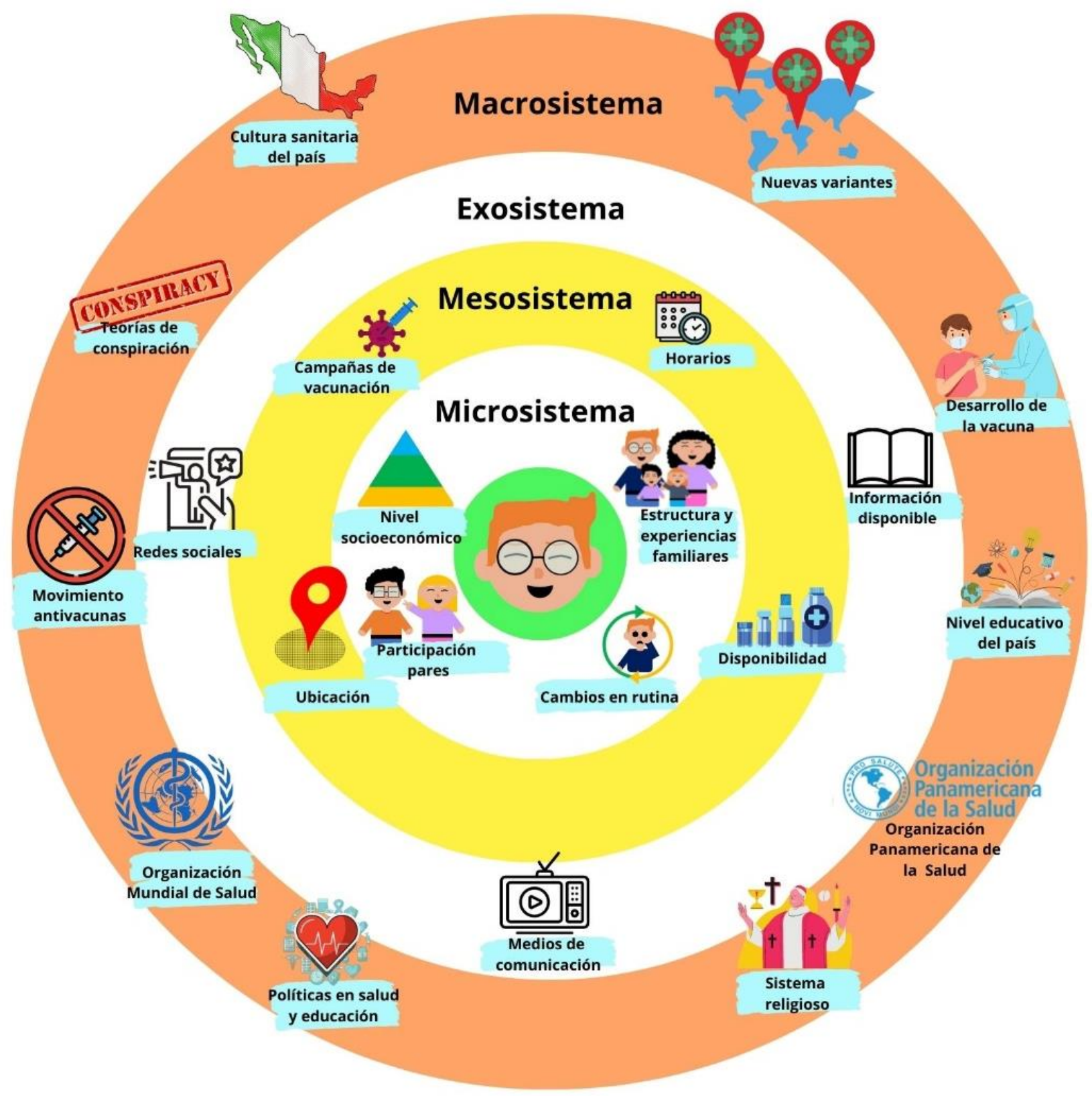

Figura 1: Modelo Ecológico de Bronfenbrenner y los factores ubicados en la vacunación versus COVID-19 Fuente: Elaboración propia.

\section{Referencias}

[1] Bonet AM, Vidal EA, Coassin R, Piva E. Acceso a la Vacuna Contra el Covid: estrategias internacionales y locales para gestionar la colisión entre el derecho humano a la salud y la economía. Cadernos Eletrônicos Direito Internacional sem Fronteiras 2021; 3 (2): 1-16.

[2]Bronfenbrenner U, Morris PA. The Ecology of Developmental Process. En: Damon W, Lerner RM, editors, Handbook of Child Psychology: Theoretical models of human development. 6th ed. Estados Unidos: John Wiley \& Sons Inc; 1998: pp. 993-1028.

[3] Castillo N. Coronavirus. Por qué hay gente que no quiere vacunarse [Internet]. CienciaUNAM. 2021 [citado 15 septiembre 2021].
Disponible en: http://ciencia.unam.mx/leer/1159/coronavirus-por-quehay-gente-que-no-quiere-vacunarse-

[4]Perspectivas culturales sobre la vacunación [Internet]. Historyofvaccines.Org. 2018 [citado 30 septiembre 2021]. Disponible en:

https://ftp.historyofvaccines.org/multilanguage/es/contenido/articulos/ perspectivas-culturales-sobre-la-vacunaci\%C3\%B3n

[5]Gestión de la infodemia sobre la COVID-19: Promover comportamientos saludables y mitigar los daños derivados de la información incorrecta y falsa [Internet]. Organización Panamericana de Salud (OPS). 2020 [citado 30 septiembre 2021]. Disponible en: https://www.who.int/es/news/item/23-09-2020-managing-the-covid19-infodemic-promoting-healthy-behaviours-and-mitigating-the-harmfrom-misinformation-and-disinformation 